\title{
PREVALENCE AND PSYCHOLOGICAL STRESS IN RECURRENT APHTHOUS STOMATITIS AMONG FEMALE DENTAL STUDENTS IN SAUDI ARABIA
}

\author{
MUHAMMED AJMAL, LUJAIN IBRAHIM, NADA MOHAMMED, \\ HEBA AL-QARNI
}

\author{
College of Dentistry-King Khalid University, Abha, Saudi Arabia
}

\begin{abstract}
Introduction. Recurrent Aphthous Stomatitis (RAS) is a common oral mucosal disorder which is characterized by recurrent ulcerations mainly confined to nonkeratinized oral mucosa. Though the exact etiology is imprecise, stress and anxiety were found to be linked with the onset of RAS. The present study was directed to know the prevalence of RAS among female dental students in King Khalid University, to find out the association between RAS and psychological stress and the effectiveness of Hospital Anxiety and Depression scale (HADS) in finding out the psychological profile of RAS patients.

Method. 122 female dental students of age group 17 to 25 years were selected for the study. Subjects with relevant medical problems and deleterious habits were excluded. A questionnaire comprising general stress related questions and HAD scale were used to assess stress. Those who were giving history of ulcer were diagnosed as $R A S$ based on the clinical features. The questionnaire results were used for statistical analysis and processed.

Result. $14 \%$ of the total students were having RAS. $70 \%$ could correlate the onset of ulcer with certain specific factors; stress being the major causative agent (91\%). In HAD scale, $88 \%$ of students were having anxiety and $65 \%$ had depression; all patients with depression had anxiety.

Conclusion. The prevalence of RAS in female dental students in KKU was around $14 \%$. There is a strong relationship between psychological stress and RAS, as in most of the other studies. HAD scale alone can be used for detecting the psychological factor in RAS.
\end{abstract}

Keywords: recurrent aphthous stomatitis, RAS, aphthous stomatitis, mouth ulcer, stress related oral ulcer

\section{Introduction}

Recurrent aphthous ulceration (RAU) or recurrent aphthous stomatitis (RAS) is one of the known painful oral mucosal conditions. In spite of wide clinical and research consideration given to RAU, ulcers are not avoidable, and most of the treatment is symptomatic. Etiopathogenesis remains poorly understood despite their high prevalence [1].

Though exact etiology and pathogenesis are uncertain, several factors are considered as decisive. Factors such as nutrition, hypersensitivity, drugs, hormones, trauma, tobacco use and psychological stress have been implicated in the onset. In addition to the suggested local

Manuscript received: 04.07.2017

Accepted: 05.10.2017

Address for correspondence: drmuhammedajmal@gmail.com and systemic elements, there is also evidence of a genetic and immunopathogenic basis [1].

The role of stress that results in RAS episodes is still unclear. It is usually believed that acute psychological disorders may play a role in the development of recurrent aphthous ulcers. Onset of ulcer may be attributed to the elevated levels of salivary cortisol or reactive oxygen species in the saliva. Stress can also lead to cheek or lip bite, or aggravate actions causing injury to the oral mucosa. Stressful situations are supposed to cause a transitory rise in salivary cortisol and/or in immune regulatory activity in inflammation by increasing the quantity and activity of leukocytes [2].

Valid and reliable studies are few and thus continuing research is needed to identify the uncertain association 
between the psychological aspect and the incidence of recurrent aphthous ulcers. There is also need of a reliable scale to assess the psychological factor for defining the underlying suspected etiology. A study group of female students were selected for the present study considering the susceptibility of this group to RAS and stress. The study by Axell and Henricsson concluded that tobacco and RAS have negative correlation, and leukoedema might prevent antigenic substances entry into the epithelium [3].

\section{Material and methods}

122 female dental students were taken randomly for the study. Students' age group ranged from 17 to 25 years. Ethical clearance was taken from the college research committee, as well as consent from all the participants. All patients selected were without any relevant medical problems like diabetes mellitus, hypertension, thyroid problems etc. and deleterious habits. Questionnaire was given to the selected subjects and it included interrogations regarding the presence of frequent oral ulcers and associated factors observed by the subjects. HAD scale questionnaire was also given to the students undergoing the study to confirm the stress factor. Those who were giving history of recurrent ulcers were diagnosed as recurrent aphthous stomatitis based on the history and confirmed by the clinical features observed by a diagnosis specialist. The questionnaire inclusion criteria was those dental students having a known history of RAS and exclusion criteria were individuals with diabetes mellitus, hypertension, thyroid problem, or those who were under any medications; reports were used for statistical analysis and then the results were processed.

\section{Results}

Results are arranged in the following categories

\section{Experience of $R A S$ among female dental students}

17 out of 122 students (14\%) experienced recurrent aphthous stomatitis. Among the 17 students, all students (100\%) had frequent episode of RAS. Most of them $(82.4 \%)$ reported that ulcer lasted less than 10 days. For $11.8 \%$ of students, ulcers heal by 10-14 days. Those who were giving history of recurrent ulcers were diagnosed as RAS patients and included in the study after the clinical examination.

\section{Associated factors}

Relationship of ulcers with menstruation, food allergy, and type of food was probed. Students were asked to reveal any specific factor association with the onset of ulcers. $70.6 \%$ of the patients felt that there were some factors associated with the onset of ulcer. Most of them could correlate the beginning of ulcers with stress and anxiety. 58\% students who had experienced ulcer reported that stress was a predisposing factor for the onset of ulcer. The others noticed that anxiety could be the reason for the ulcer $(41 \%) .47 .1 \%$ of the subjects also said there was a relationship between onset of ulcer and menstruation. $52.9 \%$ patients could not correlate between menstruation and ulcer. For $42.9 \%$, ulcers occurred before menstruation; $14.3 \%$ students noticed that ulcer occurred during the menstruation; $42.9 \%$ reported that ulcer disappeared with menstruation. Any association with ulcers and specific food type was found out. $17.6 \%$ were regular users of spicy food. Salty food was preferred by $58.8 \%$ students and $29.4 \%$ students were not regular users of salty, acidic or hot spicy food.11.8\% students suffered from food allergy. $88.2 \%$ of them had no history of food allergy (Table I).

Table I. Questionnaire and response (by the rau subjects).

\begin{tabular}{|l|lc|}
\hline 1 & \multicolumn{3}{|l|}{ Regular type of food } \\
& Hot spicy food & $17.6 \%$ \\
& Salty food & $58.8 \%$ \\
& Acidic food & $0 \%$ \\
\hline 2 & \multicolumn{3}{|l|}{ Any kind of food allergy } \\
& Yes & $11.8 \%$ \\
& No & $88.2 \%$ \\
\hline 3 & Relationship between ulcer \& menstruation \\
& Yes & $47.1 \%$ \\
& No & $52.9 \%$ \\
\hline 4 & Whether noticed Associated factors \\
& Yes & $70.6 \%$ \\
& No & $20.4 \%$ \\
\hline 5 & Associated Factors \\
& Stress 58\% \\
& Anxiety $41 \%$ \\
\hline
\end{tabular}

\section{QUESTIONNAIRE} before?

1.Did you experience any type of mouth ulcer

- Yes

- No

(If Yes please answer the following questions)

a.In that time the ulcer lasts for:

- Less than 10 days

- From 10 days to 14 days

- More than 14 days

- I don't know

b.Do you have the ulcer frequently:

- Yes

- No

c. Do you feel any factors associated with the ulcers?

- Yes

- No

- If Yes, mention the factors (like stress, anxiety, anger, sadness, any type of food, menstrual cycle etc.)...............

2.When was the last time you had the ulcer

- Before one month

- Before one year

- Other(mention the time)

- I don't know

3.Do you face any problems in your menstrual period?

- My period time is regular and I have no problems 
- My period time is irregular

(a) Do you feel that there is a relationship between your oral ulcer and menstrual period?

- Yes

- No

(b) If Yes, what is the relation?

- Ulcer comes just before the period

- Ulcer comes during the period

- Ulcer goes with the period

- Or any other, mention

4.Do you have any medical problem?

- Yes

- No you have?

If yes, which of the following medical problems do

- Hypothyroidism

- Hyperthyroidism

- Iron-deficiency anemia

- Gastro intestinal diseases

- Hypertension

- Diabetic mellitus

- Asthma

- Other (mention it)

5.Do you take any medication?

- No

- Yes (mention it)

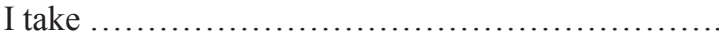

For.

6.Do you have any kind of food allergy?

- No

- Yes (mention it)

7.Do you regularly eat the following food?

- Hot spicy food

- Acidic food (soft drinks ......)

- Salty food

- Non-of the above.

8. At the time of stress or anxiety:

- I usually express my feelings (crying, talking to some body....etc.) feelings)

I usually pretend I'm ok (avoid to express your

\section{HAD scale for assessing stress factor}

HAD scale was used to assess the stress factor in ulcer patients as majority reported stress being the common predisposing factor. The Hospital Anxiety and Depression Scale (HADS) was developed to identify possible and probable cases of anxiety disorders and depression among patients in non-psychiatric hospital clinical set up.

According to HADS, 79 students out of $122(65 \%)$ were having anxiety, 42 of $122(34 \%)$ were depressed. There were 17 patients with recurrent aphthous stomatitis comprising $14 \%$ of the total subjects studied. Out of 17 students, 15 students were found having anxiety (88\%) and 11 subjects with depression (65\%) from HAD scale. All the 11 patients with depression were with anxiety as well. Only 2 subjects with recurrent aphthous stomatitis who reported to have stress factor were not shown positive results with this scale. HAD scale confirms the stress factor by showing $88 \%$ of recurrent aphthous ulcer cases diagnosed with anxiety.

\section{(HADS)}

Hospital Anxiety and Depression Scale

Patients are asked to choose one response from the four given for each interview. They should give an immediate response and be dissuaded from thinking too long about their answers. The questions relating to anxiety are marked "A", and to depression " $\mathrm{D}$ ". The score for each answer is given in the right column.

\begin{tabular}{|l|l|l|}
\hline A & I feel tense or 'wound up': & \\
\hline & Most of the time & 3 \\
\hline & A lot of the time & 2 \\
\hline & From time to time, occasionally & 1 \\
\hline & Not at all & 0 \\
\hline D & I still enjoy the things I used to enjoy: & \\
\hline & Definitely as much & 0 \\
\hline & Not quite so much & 1 \\
\hline & Only a little & 2 \\
\hline & Hardly at all & 3 \\
\hline A & I get a sort of frightened feeling as if something \\
awful is about to happen: & \\
\hline & Very definitely and quite badly & \\
\hline & Yes, but not too badly & 3 \\
\hline & A little, but it doesn't worry me & 2 \\
\hline & Not at all & 1 \\
\hline D & I can laugh and see the funny side of things: & 0 \\
\hline & As much as I always could & \\
\hline & Not quite so much now & 0 \\
\hline & Definitely not so much now & 1 \\
\hline & Not at all & 2 \\
\hline A & Worrying thoughts go through my mind: & 3 \\
\hline & A great deal of the time & \\
\hline & A lot of the time & 3 \\
\hline & From time to time, but not too often & 2 \\
\hline & Only occasionally & 1 \\
\hline D & I feel cheerful: & 0 \\
\hline & Not at all & \\
\hline & Not often & 3 \\
\hline & Sometimes & 2 \\
\hline & Most of the time & 1 \\
\hline A & I can sit at ease and feel relaxed: & 0 \\
\hline & Definitely & 0 \\
\hline & Usually & 1 \\
\hline & Not Often & 2 \\
\hline & Not at all & 3 \\
\hline
\end{tabular}




\begin{tabular}{|l|l|l|}
\hline D & I feel as if I am slowed down: & \\
\hline & Nearly all the time & 3 \\
\hline & Very often & 2 \\
\hline & Sometimes & 1 \\
\hline & Not at all & 0 \\
\hline A & $\begin{array}{l}\text { I get a sort of frightened feeling like 'butterflies' in } \\
\text { the stomach: }\end{array}$ & \\
\hline & Not at all & 0 \\
\hline & Occasionally & 1 \\
\hline & Quite Often & 2 \\
\hline & Very Often & 3 \\
\hline D & I have lost interest in my appearance: & \\
\hline & Definitely & 3 \\
\hline & I don't take as much care as I should & 2 \\
\hline & I may not take quite as much care & 1 \\
\hline & I take just as much care as ever & 0 \\
\hline A & I feel restless as I have to be on the move: & \\
\hline & Very much indeed & 3 \\
\hline & Quite a lot & 2 \\
\hline & Not very much & 1 \\
\hline & Not at all & 0 \\
\hline D & I look forward with enjoyment to things: & \\
\hline & As much as I ever did & 0 \\
\hline & Rather less than I used to & 1 \\
\hline & Definitely less than I used to & 2 \\
\hline & Hardly at all & 3 \\
\hline A & I get sudden feelings of panic: & \\
\hline & Very often indeed & 3 \\
\hline & Quite often & 2 \\
\hline & Not very often & 1 \\
\hline & Not at all & 0 \\
\hline D & I can enjoy a good book or radio or TV program: & \\
\hline & Often & 0 \\
\hline & Sometimes & 2 \\
\hline & Not often & 3 \\
\hline & Very seldom & \\
\hline & \multicolumn{2}{|l|}{} \\
\hline & $\mathbf{0 - 7}=$ Normal & \\
\hline & $\mathbf{8 - 1 0}=$ Borderline abnormal & \\
\hline & $\mathbf{1 1 - 2 1}=$ Abnormal & \\
\hline
\end{tabular}

\section{Discussion}

Recurrent aphthous stomatitis is one of the most common painful oral mucosal conditions seen among patients. These ulcers usually start first in childhood or adolescence. Appearance of round to ovoid circumscribed ulcers with yellowish or gray floor and anerythematous halo with recurring nature is characteristic of RAU. Prevalence of recurrent aphthous ulcer varies between 5-60\% depending on the group examined [4].

The largest study on RAU was on 10,000 young adult patients from 21 different countries. The study demonstrated that $38.7 \%$ and $49.7 \%$ of male and female patients respectively suffered from RAU in their lives.
Approximately $25 \%$ of the patients had an experience of episode in the last 12 months [5].

The definitive etiology and pathogenesis of RAU is still an enigma. Nutritional factors, drugs like NSAID, microbial agents, physical injury, tobacco, allergy to certain food, hormones, and psychological stress are found to play a fundamental role [6]. High frequency and decreased quality of life due to the recurring painful ulcers have resulted in a great deal of research into the etiology and efficient therapy.

Our study could find association with psychological stress, anxiety and a relation with onset of ulcer with menstruation. Most of the studies could show that there is no established association between aphthous stomatitis and premenstrual period, pregnancy, or menopause [7]. On the other hand, the study by Tony Axell et al noted that menstruation is one of the common triggering factors [8]. The correlation between the hormonal level and RAU needs further future studies.

A positive association between RAU and patient's psychological status has been frequently described. The study by Albanidou-Farmaki et al. presented that anxiety could be a reason for RAU. This was based on the result of significant differences of anxiety level noted between patients with RAU and control subjects [2].

Soto-Araya et al. reported that unlike depression, anxiety and stress can significantly be related to onset of RAU [9]. Cohen also found a high incidence of RAU in those who are under stressful conditions [10].

Mahmoud $\mathrm{K}$ et al. could not find a significant difference in ulcers between the patients and controls according to the depressive state [11].

Mahmoud et al. assessed personality and psychological profile using HAD and NeurocriticismExtaversion-openness Five Factor Inventory (NEO-FFI). Females had higher HAD anxiety score in both controls and patients. There was no correlation between intensity, duration and frequency of ulcer with HAD score. There was no relation to depression though higher level of anxiety was recorded. It was also noted that stressful situation is related to onset of ulcers rather than personality profiles and stable psychological traits [12].

The study by Andrews et al showed that relaxation/ imagery treatment can significantly reduce the occurrence of ulcers which is again favoring the stress factor [13].

It is suggested that stress, with its presumed effects on the immune system, constitutes one of the major causative agents of RAS [2]. An insight into a patient's psychological status can be estimated from both serum free and salivary cortisol levels [14].

Cortisol, otherwise known as stress hormone, has been the indicator for the evaluation of stress. The assessment of cortisol in saliva has gained interest in studies for evaluating anxiety. Salivary cortisol may actually provide a better measure than serum cortisol, as 
it is a more accurate measure of the amount of unbound cortisol compared to serum. Diurnal variation and circadian rhythm of both salivary cortisol and plasma cortisol are very similar [15].

Many studies have suggested that psychological disturbances such as stress and anxiety could play a role in the onset and recurrence of RAS lesions. However, the obtained results were rather varied. Pedersen and Picek et al. evaluated psychological stress in patients with RAU and concluded that there was no relationship between psychological life stress and repetitive RAU $[16,17]$.

Therefore, the aim of this study was to conduct an investigation on female subjects, the influence of various factors on the onset of ulcers. Stress, anxiety, and hormonal changes were the particular factors the subjects could correlate with recurrence of the ulcers. Stress factor was confirmed using a validated questionnaire that appraised the patients' level of stress by judging the presence, frequency and intensity of stress symptoms. Based on the score, subjects were divided as normal, borderline abnormal and abnormal.

Significant number of RAU patients had psychological stress confirmed by HAD scale. This perceived information supports the role of stress as a modifier of RAS. $65 \%$ of subjects also had depression, though previous studies have shown that there is no correlation on onset of ulcers in patients with depression. Based on our study results, it can be suggested that stress may play a vital role in the occurrence of ulcers, along with genetic and immunopathologic basis. The results direct for future studies to evaluate the influence of psychotherapy on RAS onset and symptoms. The lack of a direct association between the level of stress and severity of RAS episodes suggests that psychological stress may act as a trigger or modifying factor rather than an etiological factor in susceptible RAS patients [18].

HADS was found to perform well in assessing the symptom and severity of anxiety disorders and depression in somatic, psychiatric, primary care patients and in the general population [19].

Review of literature revealed that HADS, though it is concise, displayed comparable sensitivity and specificity as longer versions of General Health Questionnaire (GHQ). When matched to other questionnaires for anxiety and depression in common use such as Beck Depression Inventory (BDI), State Trait Anxiety Index (STAI), Clinical Anxiety Scale (CAS), and Symptom Checklist 90 (SCL90) and Anxiety and Depression subscales, the correlations to Hospital Anxiety and Depression Scale-Depression (HADS-D) and Hospital Anxiety and Depression ScaleAnxiety (HADS-A), respectively, were between 0.60 and 0.80 , which can be considered as medium to strong correlations. HADS-D was also comparable to Montgomery Asberg Depression Rating Scale [19].

It can be suggested that HAD scale is effective for identifying the psychological aspect of recurrent aphthous stomatitis, as most of the subjects with stress were diagnosed having anxiety or depression correlating to the questionnaire. Limitation of present study is not including the correlation of severity of RAS and HADS rating which would confirm the predictor.

\section{Conclusion}

Because of the vague etiology and symptomatic treatment available, research and evidence based studies on RAU still continue and the present data can be considered as noteworthy. The usefulness of psychotherapy on RAS symptoms and onset can be explored based on the strong valid results obtained from the study. HAD scale can be used to assess the anxiety and depression efficiently to identify the underlying modifying factors.

\section{References}

1. Ship JA. Recurrent aphthous stomatitis: An update. Oral Surg Oral Med Oral Pathol Oral Radiol Endod. 1996;81(2):141-147.

2. Albanidou-Farmaki E, Poulopoulos AK, Epivatianos A, Farmakis K, Karamouzis M, Antoniades D. Increased anxiety level and high salivary and serum cortisol concentrations in patients with recurrent aphthous stomatitis. Tohoku J Exp Med. 2008;214:291-296.

3. Axéll T, Henricsson V. Association between recurrent aphthous ulcers and tobacco habits. Scand J Dent Res. 1985;93:239-242.

4. Jurge S, Kuffer R, Scully C, Porter SR. Mucosal disease series. Number VI. Recurrent aphthous stomatitis. Oral Dis. 2006;12:1-21.

5. Embil JA, Stephens RG, Manuel FR. Prevalence of recurrent herpes labialis and aphthous ulcers among young adults on six continents. Can Med Assoc J. 1975;113:627-630.

6. Porter SR, Scully C, Pedersen A. Recurrent aphthous stomatitis. Crit Rev Oral Biol Med. 1998;9(3):306-321.

7. McCartan BE, Sullivan A. The association of menstrual cycle, pregnancy, and menopause with recurrent oral aphthous stomatitis: a review and critique. Obstet Gynecol. 1992;80(3):455-458.

8. Axéll T, Henricsson V. The occurrence of recurrent aphthous ulcers in an adult Swedish population. Acta Odontol Scand. 1985;43(2):121-125.

9. Soto Araya M, Rojas Alcayaga G, Esguep A. Association between psychological disorders and the presence of Oral lichen planus, Burning mouth syndrome and Recurrent aphthous stomatitis. Med Oral. 2004;9:1 7.

10. Cohen L. Etiology, pathogenesis and classifications of aphthous stomatitis and Behçet's syndrome. J Oral Pathol. 1978; 7:347-352.

11. Varkal MD, Uyar ET, Bilal N, Akbulut T, Aydin EP. Anxiety levels and parental bonding in recurrent aphthous stomatitis patients. Bulletin of Clinical Psychopharmacology. 2015;25:S45-S46.

12. AL-Omiri MK, Karasneh J, Lynch E. Psychological profiles in patients with recurrent aphthous ulcers. Int J Oral Maxillofac Surg. 2012;41(3):384-388.

13. Andrews VH, Hall HR. The effects of relaxation/imagery training on recurrent aphthous stomatitis: a preliminary study. Psychosom Med. 1990;52(5):526-535.

14. McCartan BE, Lamey PJ, Wallace AM. Salivary cortisol 


\section{Original Research}

and anxiety in recurrent aphthous stomatitis. J Oral Pathol Med. 1996;25:357 359 .

15. Safarzadeh E, Mostafavi F, Haghi Ashtiani MT. Determination of salivary cortisol in healthy children and adolescents. Acta Med Iran. 2005;43:32-36.

16. Pedersen A. Psychologic stress and recurrent aphthous ulceration. J Oral Pathol Med. 1989;18:119-122.

17. Picek P, Buljan D, Rogulj AA, Stipetić-Ovcarićek J, Catić
A, Plestina S, et al. Psychological status and recurrent aphthous ulceration. Coll Antropol. 2012;36:157-159.

18. Gallo Cde B, Mimura MA, Sugaya NN. Psychological stress and recurrent aphthous stomatitis. Clinics (Sao Paulo). 2009;64(7):645-648.

19. Bjelland I, Dahl AA, Haug TT, Neckelmann D. The validity of the Hospital Anxiety and Depression Scale: An updated literature review. J Psychosom Res. 2002;52(2):69-77. 